\title{
Fetal Ventriculomegaly: A Review of Literature
}

\author{
Review began 02/05/2022 \\ Review ended 02/08/2022 \\ Published 02/18/2022 \\ ๑) Copyright 2022 \\ Alluhaybi et al. This is an open access \\ article distributed under the terms of the \\ Creative Commons Attribution License CC- \\ BY 4.0., which permits unrestricted use, \\ distribution, and reproduction in any \\ medium, provided the original author and \\ source are credited.
}

\author{
Abdulelah A. Alluhaybi ${ }^{1}$, Khalid Altuhaini $^{1}$, Maqsood Ahmad ${ }^{1}$ \\ 1. Pediatric Neurosurgery, King Fahad Medical City/National Neuroscience Institute, Riyadh, SAU \\ Corresponding author: Abdulelah A. Alluhaybi, aaallehibi@gmail.com
}

\begin{abstract}
Fetal ventriculomegaly refers to ventricular enlargement that is diagnosed prenatally. It is one of the most common fetal anomalies. The diagnosis is made by ultrasound when the arterial diameter of the ventricle is more than $10 \mathrm{~mm}$. Once it is diagnosed, further evaluation by detailed ultrasound, fetal MRI, and genetic studies is required. Prenatal surgical management of fetal ventriculomegaly is still limited and associated with high risks. Postnatal management is similar to the treatment of other types of hydrocephalus. Fetal ventriculomegaly is a heterogeneous condition with various etiologies and a wide spectrum of neurodevelopmental outcomes. The outcomes depend mainly on the severity of ventriculomegaly and associated structural abnormalities. This article aims to review the literature about various aspects of fetal ventriculomegaly.
\end{abstract}

\section{Categories: Neurosurgery}

Keywords: fetal ventriculomegaly, hydrocephalus, genetics, fetal ultrasound, fetal mri, cerebrospinal fluid, ventricular shunts

\section{Introduction And Background}

Fetal ventriculomegaly is a ventricular dilatation that is detected on ultrasonography prenatally [1]. It occurs in two per 1000 live births $[2,3]$ and is considered one of the most common fetal anomalies detected on ultrasonography during the second trimester [4]. It is categorized as mild (10-12 $\mathrm{mm})$, moderate (13$15 \mathrm{~mm})$, or severe ( $\geqslant 16 \mathrm{~mm}$ ) according to ventricular diameter, measured on antenatal sonography or in utero MRI [5]. Fetal ventriculomegaly has multiple causes and produces a broad spectrum of neurodevelopmental outcomes [4]. Once it is identified, detailed ultrasonography should be performed to rule out other fetal structural anomalies [1]. Severe ventriculomegaly usually has obstructive causes and is associated with hydrocephalus [1]. Mild ventriculomegaly is often found incidentally and is considered benign, but it can be associated with genetic or structural abnormalities [1]. Isolated ventriculomegaly is defined as ventriculomegaly that is not associated with structural or genetic abnormalities identified on ultrasonography [6].

Postnatal management depends mainly on the rate of progression of ventriculomegaly and the development of hydrocephalus [4]. Neurodevelopmental outcomes and prognosis are influenced mainly by the severity of both ventriculomegaly and underlying structural or genetic abnormalities [7]. We review the causes, diagnosis, and treatment of fetal ventriculomegaly and the prognosis of affected patients.

\section{Review}

The differential diagnosis of fetal ventriculomegaly is broad and includes normal variants when the size of the ventricle approaches $10 \mathrm{~mm}[1,8]$. Fetal ventriculomegaly may be considered an indicator of chromosomal abnormalities, infection, or cerebral malformation, particularly when it is isolated [9]. Therefore, an extensive evaluation is necessary to determine the size of the ventricle and the underlying cause.

\section{Etiology}

The causes of fetal ventriculomegaly are divided into three main categories: cerebral parenchymal loss; obstructive causes; and overproduction of cerebrospinal fluid (CSF) [10]. Although isolated ventriculomegaly may be benign, chromosomal abnormalities are found in $2 \%-12 \%$ of cases [11]. Different causative processes include abnormal turnover of CSF, neuronal and migration disorders, and infection. Some cases are inherited X-linked or, in rare cases, autosomal recessive traits [9]. Intrauterine infections are found in approximately $5 \%$ of patients with fetal ventriculomegaly, and Toxoplasma gondii, rubella, cytomegalovirus, and herpes simplex virus infections are found in $10 \%-20 \%$ of those with severe isolated ventriculomegaly [12]. The most common structural causes of fetal ventriculomegaly include aqueductal stenosis, Chiari malformation type II, dysgenesis of the corpus callosum, and abnormalities of the posterior fossa $[13,14]$. Other causes include intraventricular hemorrhage and cortical development disorders, and other associated disorders include hydranencephaly and holoprosencephaly [15].

\section{Diagnosis}


Neuroimaging, including fetal ultrasonography and MRI, is crucial for the diagnosis of fetal ventriculomegaly. Each imaging modality has advantages and disadvantages [16]. When fetal ventriculomegaly is identified on screening antenatal ultrasonography, further workup with fetal ultrasonography or MRI is usually required [16].

\section{Fetal ultrasonography}

At the gestational age of 13-14 weeks, the lateral ventricles can be recognized; they change with the subsequent development of the cerebrum, and the choroid plexus usually serves as the landmark [17]. Although the overall ventricular diameter increases over time, the atrium remains stable during the second and third trimesters; for this reason, these are the preferred times for measurement [18]. The normal ventricular diameter in the fetal brain remains between 4.5 and $7.6 \mathrm{~mm}$ during the gestational ages of 15 and 40 weeks [8]. Most international guidelines mandate antenatal ultrasonography during the second trimester for measuring the diameter of the lateral ventricle in the axial transventricular plane. A diameter of $\geqslant 10 \mathrm{~mm}$ with at least 2.5 standard deviations above normal indicates ventriculomegaly and is considered abnormal [19].

\section{Fetal MRI}

Fetal MRI is an excellent diagnostic modality that enables the detection of additional abnormalities in approximately $50 \%$ of ventriculomegaly cases [20], such as agenesis of the corpus callosum, absence of the septum pellucidum, disorders of cortical development, and cerebrovascular abnormalities [21]. It is more sensitive than ultrasonography for detecting cortical malformation, hemorrhage, and parenchymal disorders [22]. Not all these findings may be clinically significant or associated with a poor prognosis, but early identification is helpful for parent counseling and directs further follow-up [23,24]. Measurements of ventricular diameter on axial MRI and ultrasonography differ by 1-2 $\mathrm{mm}$ [25]; however, measurements on coronal MRI and ultrasonography are highly similar [26].

\section{Genetic testing}

Karyotyping, used to identify aneuploidy and chromosomal abnormalities, is the standard method of genetic evaluation of fetal structural anomalies. An abnormal karyotype has been found in approximately $5 \%$ of cases of mild to moderate ventriculomegaly; trisomy 21 is the most common. Abnormal results of chromosomal microarray have been found in $10 \%$ of fetuses with abnormal findings on ultrasonography [27]. In 6\% of fetuses with structural anomalies and normal karyotypes, microarray analysis has revealed clinically significant information [28]. Chromosomal microarray analysis is recommended as the first-tier test because it can reveal submicroscopic chromosomal abnormalities that are undetectable in conventional karyotyping [29].

\section{Prenatal management}

In the prenatal management of fetal ventriculomegaly, the efficacy of intrauterine ventricular shunting procedures is still limited [30]. In 1982, the International Fetal Medicine and Surgery Society established a voluntary international registry of fetal surgery. Thirty-nine cases of prenatal shunting have been reported; the fetal survival rate is $83 \%$. Neurological disability has been severe and mild to moderate in $>50 \%$ and $12 \%$ of the survivors, respectively; $35 \%$ have exhibited normal development [31-33]. Although early prenatal CSF procedures had unfavorable outcomes, the use of fetal CSF shunting has increased as a result of improvement in prenatal diagnosis and advances in fetal surgery [34].

\section{Postnatal management}

The first step in management is to identify the underlying cause and determine whether hydrocephalus is present. If hydrocephalus is present, early delivery and management could be beneficial; however, this protocol depends on maturation of the lungs and stability of the fetus. Postnatal management depends mainly on the cause of the ventriculomegaly. Only patients with obstructive causes and progressive hydrocephalus are candidates for CSF diversion procedures. Conservative measures can be tried in patients whose ventricular diameter is stable and who have no evidence of increased intracranial pressure. The indications for and timing of surgical intervention depend mainly on two factors: (1) the degree and progression of ventriculomegaly, according to findings of clinical examination and neuroimaging follow-up, and (2) the presence or absence of increased intracranial pressure. Surgical treatment of ventriculomegaly in fetuses is similar to that in cases diagnosed after birth and includes different types of CSF reservoir devices, shunts, and endoscopic fenestration [35].

\section{Prognosis}

The outcome in affected fetuses depends mainly on the severity of ventriculomegaly and associated anomalies. In isolated ventriculomegaly, the size of the ventricles influences overall outcome, mild isolated ventriculomegaly carries the most favorable prognosis [7,36]. In a study of 176 patients with ventriculomegaly, Gaglioti et al. found that the survival rates were $98 \%$, $80 \%$, and $33 \%$ in mild, moderately severe, and severe cases, respectively. The worse outcomes in severe cases are related to underlying 
structural abnormalities found in $75 \%$ of such cases [37]. In a meta-analysis, Pagani et al. found that neurodevelopmental delay was present in only $8 \%$ of patients with mild to moderate ventriculomegaly [7]. Conversely, neurodevelopment was normal in only $5 \%-8 \%$ of patients with severe ventriculomegaly [37-39].

\section{Conclusions}

Fetal ventriculomegaly has multiple causes and the developmental outcomes vary. Neuroimaging, including fetal ultrasonography and MRI, help diagnose fetal ventriculomegaly. When it is diagnosed, chromosomal microarray analysis is recommended because it can reveal submicroscopic chromosomal abnormalities that are undetectable in conventional karyotyping. Prenatal management of fetal ventriculomegaly is still limited, and in cases diagnosed both prenatally and after birth, postnatal management includes the use of different types of CSF reservoir devices, shunts, and endoscopic fenestration. The outcome of fetal ventriculomegaly depends mainly on the severity of both ventriculomegaly and any associated anomalies that may be present.

\section{Additional Information \\ Disclosures}

Conflicts of interest: In compliance with the ICMJE uniform disclosure form, all authors declare the following: Payment/services info: All authors have declared that no financial support was received from any organization for the submitted work. Financial relationships: All authors have declared that they have no financial relationships at present or within the previous three years with any organizations that might have an interest in the submitted work. Other relationships: All authors have declared that there are no other relationships or activities that could appear to have influenced the submitted work.

\section{References}

1. Society for Maternal-Fetal Medicine, Fox NS, Monteagudo A, Kuller JA, Craigo S, Norton ME: Mild fetal ventriculomegaly: diagnosis, evaluation, and management. Am J Obstet Gynecol. 2018, 219:B2-9. 10.1016/j.ajog.2018.04.039

2. Laurence KM, Carter CO, David PA: Major central nervous system malformations in South Wales. I. Incidence, local variations and geographical factors. Br J Prev Soc Med. 1968, 22:146-60. 10.1136/jech.22.3.146

3. Edwards JH: Congenital malformations of the central nervous system in Scotland . Br J Prev Soc Med. 1958, 12:115-30. 10.1136/jech.12.3.115

4. Pisapia JM, Sinha S, Zarnow DM, Johnson MP, Heuer GG: Fetal ventriculomegaly: diagnosis, treatment, and future directions. Childs Nerv Syst. 2017, 33:1113-23. 10.1007/s00381-017-3441-y

5. Griffiths PD, Reeves MJ, Morris JE, Mason G, Russell SA, Paley MN, Whitby EH: A prospective study of fetuses with isolated ventriculomegaly investigated by antenatal sonography and in utero MR imaging. Am J Neuroradiol. 2010, 31:106-11. 10.3174/ajnr.A1767

6. Vergani P, Locatelli A, Strobelt N, Cavallone M, Ceruti P, Paterlini G, Ghidini A: Clinical outcome of mild fetal ventriculomegaly. Am J Obstet Gynecol. 1998, 178:218-22. 10.1016/S0002-9378(98)80003-3

7. Pagani G, Thilaganathan B, Prefumo F: Neurodevelopmental outcome in isolated mild fetal ventriculomegaly: systematic review and meta-analysis. Ultrasound Obstet Gynecol. 2014, 44:254-60. 10.1002/uog.13364

8. Farrell TA, Hertzberg BS, Kliewer MA, Harris L, Paine SS: Fetal lateral ventricles: reassessment of normal values for atrial diameter at US. Radiology. 1994, 193:409-11. 10.1148/radiology.193.2.7972754

9. Gaglioti P, Oberto M, Todros T: The significance of fetal ventriculomegaly: etiology, short- and long-term outcomes. Prenat Diagn. 2009, 29:381-8. 10.1002/pd.2195

10. McKechnie L, Vasudevan C, Levene M: Neonatal outcome of congenital ventriculomegaly . Semin Fetal Neonatal Med. 2012, 17:301-7. 10.1016/j.siny.2012.06.001

11. Nyberg DA, Mack LA, Hirsch J, Pagon RO, Shepard TH: Fetal hydrocephalus: sonographic detection and clinical significance of associated anomalies. Radiology. 1987, 163:187-91. 10.1148/radiology.163.1.3547493

12. Weichert J, Hartge D, Krapp M, Germer U, Gembruch U, Axt-Fliedner R: Prevalence, characteristics and perinatal outcome of fetal ventriculomegaly in 29,000 pregnancies followed at a single institution. Fetal Diagn Ther. 2010, 27:142-8. 10.1159/000304735

13. D'Addario V, Pinto V, Di Cagno L, Pintucci A: Sonographic diagnosis of fetal cerebral ventriculomegaly: an update. J Matern Fetal Neonatal Med. 2007, 20:7-14. 10.1080/14767050601036188

14. Garel C, Luton D, Oury JF, Gressens P: Ventricular dilatations. Childs Nerv Syst. 2003, 19:517-23. 10.1007/s00381-003-0795-0

15. Glenn OA, Cuneo AA, Barkovich AJ, Hashemi Z, Bartha AI, Xu D: Malformations of cortical development: diagnostic accuracy of fetal MR imaging. Radiology. 2012, 263:843-55. 10.1148/radiol.12102492

16. Nagaraj UD, Kline-Fath BM: Imaging diagnosis of ventriculomegaly: fetal, neonatal, and pediatric. Childs Nerv Syst. 2020, 36:1669-79. 10.1007/s00381-019-04365-Z

17. Filly RA, Goldstein RB, Callen PW: Fetal ventricle: importance in routine obstetric sonography. Radiology. 1991, 181:1-7. 10.1148/radiology.181.1.1887016

18. Alagappan R, Browning PD, Laorr A, McGahan JP: Distal lateral ventricular atrium: reevaluation of normal range. Radiology. 1994, 193:405-8. 10.1148/radiology.193.2.7972753

19. Sonographic examination of the fetal central nervous system: guidelines for performing the 'basic examination' and the 'fetal neurosonogram'. Ultrasound Obstet Gynecol. 2007, 29:109-16. 10.1002/uog.3909

20. Morris JE, Rickard S, Paley MN, Griffiths PD, Rigby A, Whitby EH: The value of in-utero magnetic resonance imaging in ultrasound diagnosed foetal isolated cerebral ventriculomegaly. Clin Radiol. 2007, 62:140-4. 
10.1016/j.crad.2006.06.016

21. Griffiths PD, Brackley K, Bradburn M, et al.: Anatomical subgroup analysis of the MERIDIAN cohort: ventriculomegaly. Ultrasound Obstet Gynecol. 2017, 50:736-44. 10.1002/uog.17475

22. Li Y, Estroff JA, Mehta TS, et al.: Ultrasound and MRI of fetuses with ventriculomegaly: can cortical development be used to predict postnatal outcome?. AJR Am J Roentgenol. 2011, 196:1457-67. 10.2214/AJR.10.5422

23. Damaj L, Bruneau B, Ferry M, et al.: Pediatric outcome of children with the prenatal diagnosis of isolated septal agenesis. Prenat Diagn. 2010, 30:1143-50. 10.1002/pd.2628

24. Scelsa B, Rustico M, Righini A, et al.: Mild ventriculomegaly from fetal consultation to neurodevelopmental assessment: a single center experience and review of the literature. Eur J Paediatr Neurol. 2018, 22:919-28. 10.1016/j.ejpn.2018.04.001

25. Levine D, Trop I, Mehta TS, Barnes PD: MR imaging appearance of fetal cerebral ventricular morphology . Radiology. 2002, 223:652-60. 10.1148/radiol.2233011336

26. Garel C, Alberti C: Coronal measurement of the fetal lateral ventricles: comparison between ultrasonography and magnetic resonance imaging. Ultrasound Obstet Gynecol. 2006, 27:23-7. 10.1002/uog.2666

27. Donnelly JC, Platt LD, Rebarber A, Zachary J, Grobman WA, Wapner RJ: Association of copy number variants with specific ultrasonographically detected fetal anomalies. Obstet Gynecol. 2014, 124:83-90. 10.1097/AOG.0000000000000336

28. Wapner RJ, Martin CL, Levy B, et al.: Chromosomal microarray versus karyotyping for prenatal diagnosis . N Engl J Med. 2012, 367:2175-84. 10.1056/NEJMoa1203382

29. Toren A, Alpern S, Berkenstadt M, Bar-Yosef O, Pras E, Katorza E: Chromosomal microarray evaluation of fetal ventriculomegaly. IMAJ. 2020, 22:639-44.

30. Bruner JP, Davis G, Tulipan N: Intrauterine shunt for obstructive hydrocephalus--still not ready . Fetal Diagn Ther. 2006, 21:532-9. 10.1159/000095668

31. Harrison MR, Filly RA, Golbus MS, et al.: Fetal treatment 1982. N Engl J Med. 1982, 307:1651-2. 10.1056/NEJM198212233072623

32. Manning FA, Harrison MR, Rodeck C, Members of the International Fetal Medicine and Surgery Society: Catheter shunts for fetal hydronephrosis and hydrocephalus . N Engl J Med. 1986, 315:336-40. 10.1056/NEJM198607313150532

33. von Koch CS, Gupta N, Sutton LN, Sun PP: In utero surgery for hydrocephalus. Childs Nerv Syst. 2003, 19:574-86. 10.1007/s00381-003-0775-4

34. Peiro JL, Fabbro MD: Fetal therapy for congenital hydrocephalus-where we came from and where we are going. Childs Nerv Syst. 2020, 36:1697-712. 10.1007/s00381-020-04738-9

35. Wang KC, Lee JY, Kim SK, Phi JH, Cho BK: Fetal ventriculomegaly: postnatal management. Childs Nerv Syst. 2011, 27:1571-3. 10.1007/s00381-011-1556-0

36. Cardoen L, De Catte L, Demaerel P, Devlieger R, Lewi L, Deprest J, Claus F: The role of magnetic resonance imaging in the diagnostic work-up of fetal ventriculomegaly. Facts Views Vis Obgyn. 2011, 3:159-63.

37. Gaglioti P, Danelon D, Bontempo S, Mombrò M, Cardaropoli S, Todros T: Fetal cerebral ventriculomegaly: outcome in 176 cases. Ultrasound Obstet Gynecol. 2005, 25:372-7. 10.1002/uog.1857

38. Graham E, Duhl A, Ural S, Allen M, Blakemore K, Witter F: The degree of antenatal ventriculomegaly is related to pediatric neurological morbidity. J Matern Fetal Med. 2001, 10:258-63. 10.1080/714052753

39. Breeze AC, Alexander PM, Murdoch EM, Missfelder-Lobos HH, Hackett GA, Lees CC: Obstetric and neonatal outcomes in severe fetal ventriculomegaly. Prenat Diagn. 2007, 27:124-9. 10.1002/pd.1624 